\title{
Maintaining Source Privacy under Eavesdropping and Node Compromise Attacks
}

\author{
Kanthakumar Pongaliur and Li Xiao \\ Computer Science and Engineering \\ Michigan State University, \\ East Lansing, Michigan, U.S.A. \\ Email: \{pongaliu, 1xiao\}@cse.msu.edu
}

\begin{abstract}
In a sensor network, an important problem is to provide privacy to the event detecting sensor node and integrity to the data gathered by the node. Compromised source privacy can inadvertently leak event location. Existing techniques use either random walk path or generate fake event packets to make it hard for the adversary to traceback to the source, since encryption alone may not help prevent a traffic analysis attack. In this work, without using the traditional overhead intensive methods, we present a scheme to hide source information using cryptographic techniques incurring lower overhead. The packet is modified en route by dynamically selected nodes to make it difficult for a malicious entity to traceback the packet to a source node and also prevent packet spoofing. This is important because the adversary model considers a super-local eavesdropper having the ability to compromise sensor nodes. We analyze the ability of our proposed scheme to withstand different attacks and demonstrate its efficiency in terms of overhead and functionality when compared to existing work.
\end{abstract}

\section{INTRODUCTION}

Sensor networks have significant salience to different fields, ranging from military applications to personal health monitoring. The enormity and popularity of sensor network applications is aided by the form factor and the cost of the sensor node. Although, it does induce certain limitations like low processing power and battery life, the form factor (smaller size) helps the sensor node to be inconspicuous in the sensing area and is useful for tracking events without being recognized. The sensor nodes use wireless transmissions, which is out in the open and can be overheard in the communication vicinity. Without precaution, a malicious entity overhearing packet transmission can traceback the packet to the source. This can lead to the position of the source being revealed along with the location and time of event occurrence. Considering a mission critical military application or a simple environmental application, any leakage of information such as event location or time can prove beneficial to the adversary and costly to the network goal. Hence, irrespective of the application type, privacy of the monitored event holds importance which in turn requires providing privacy to source nodes.

Source privacy is generally compromised by the meta or contextual information of a packet, and not by the actual content of the packet. This has led many researchers to note that, source privacy cannot be addressed by encryption alone. A significant amount of work guided towards using some form of simulating the source [2], [4], [6], [11], [15] or using a random walk [2], [12] has been performed to guarantee source privacy. The primary drawback of these approaches is the amount of overhead incurred to simulate a source or to redirect traffic randomly. Additionally, the studies so far only consider eavesdropping (mostly local, but some global) adversaries, while a malicious adversary that can easily compromise nodes is not considered as part of the threat model. We consider a strong threat model, in which the adversary compromises nodes while able to eavesdrop over the communication network. The adversary has access to all the cryptographic elements of the compromised node.

This paper aims to maintain source privacy under eavesdropping and node compromise attacks (SPENA). The contribution of the paper is as follows. We present an encryption based method to compliment other techniques to increase source privacy. We use a one-way hash chain based keying mechanism to hide the source information. The one-way hash function generates a series of one-time use keys. This is further used to obfuscate an additional partial hash by dynamically selected nodes preventing a traceback by the adversary. The threat model considered allows the adversary to super-locally eavesdrop, while also being able to compromise nodes. In super-local eavesdropping, the adversary eavesdrops over a local area but can overhear communication over significantly larger coverage area than a sensor node. An example of such an adversary is a laptop class attacker. When a node is compromised, the adversary has access to all the cryptographic information available to the node. We also consider the case, in which the adversary has access to data packets of past communications, stored at the compromised node. A detailed analysis of SPENA is provided and a comprehensive comparison with existing schemes is presented to show its effectiveness and efficiency.

The paper is organized as follows. Section II details the existing work on source privacy. In Section III, we present the problem statement. Next, we describe the SPENA scheme in Section IV. This is followed by the analysis and evaluation in Section V. Finally, we have the conclusion in Section VI.

\section{RELATED WORK}

There has been a lot of work to incorporate the security principles in sensor network. Protocols like TinySec [3], TinyPK [13], SERP [7] have been developed to provide security and 
to maintain integrity of data. In [10], Roosta, et al. presented a taxonomy of attacks on sensor networks and emphasized how traffic analysis attack can leak source information.

Privacy in sensor networks has been studied in detail with some researchers giving significance to hiding the basestation while others providing source privacy. Kamat, et al. developed a phantom routing technique for flooding as well as singlepath routing [2]. They were among the earlier groups to research source privacy and present multiple techniques to guarantee the same. First technique uses fake sources, with nodes sending fake event packets to confuse the adversary. The second technique called phantom routing, involves taking a random walk before forwarding the packet towards the basestation in an attempt to increase the complexity of the adversary to backtrack to the source. Although the schemes are robust, they have a large overhead involved and may not withstand attacks under a collaborative adversary model.

In [4], Mehta, et al. similarly present two techniques; namely periodic collection and source simulation to wade off global eavesdropping attack. The source simulation technique is similar to the fake sources technique presented in [2]. In periodic collection, each node reports back to the basestation periodically, irrespective of whether it detects an event or not. The weakness in case of periodic collection type technique is the latency incurred as well as overhead, while in source simulation, it is the overhead involved.

Wang et al. [12] present a privacy-aware parallel routing scheme to maximize the source traceback time for adversary. The event packets from the same source are routed over different paths to the basestation. Additionally, a weighted random stride routing is proposed that breaks the entire routing into strides. Although it is a nice scheme, one of the restrictions is the requirement of knowledge of sensor locations to know the forwarding angle. Unless a method to deduce the angle is provided, it becomes a special case of random walk technique discussed by Kamat et al. [2]. Also, the parallel routing scheme will not be effective in protecting source privacy in case of a global eavesdropping adversary.

In [5], Nezhad, et al. present an exhaustive label switching based protocol to provide source and basestation anonymity. One of the drawbacks is the requirement of the availability of global network information to the basestation. With the knowledge of the topology of the network, the sink constructs a routing tree (sink as the root) with each link having a separate label. When a node receives a packet, it switches the label of the packet to the upstream link and the packet gets propagated. Other than the basestation requiring global knowledge of the network, each node needs to perform detailed processing and reconstruction of the packets passing through them.

Shao, et al. present a statistically strong source anonymity scheme for sensor networks [11]. The authors use a exponentially distributed dummy traffic generation scheme called FitProbRate. The scheme differs from other similar works such that the dummy traffic is not generated at a constant rate but at a dynamic rate decided by the Fitprob parameter. This scheme is a vast improvement over source simulation and fake sources, but again has the drawback of having overhead due to dummy packet generation even though it will not be as much as source simulation presented in [4].

All the works discussed so far only consider a passive adversary. Most consider a local eavesdropping adversary while some provide solution to a global eavesdropping adversary. In SPENA, we consider an eavesdropping adversary having node compromise capabilities. We present a packet altering scheme, which has lesser overhead compared to a source simulation or random walk scheme. Also, when compared to a label switching scheme like [5], SPENA does not need every node on the path to perform packet transformation and does not need the basestation to be aware of the network topology. The comparison is detailed in Section V.

\section{Problem Statement}

In this section, we begin by describing the sensor network model. Then, we present a threat model with the adversary having super-local eavesdropping capability, and the ability to compromise a limited number of sensor nodes. Based on the network and the threat model, we define our problem statement.

\section{A. Network Model}

The sensor network is a homogeneous network of sensor nodes spread over a vast area. The sensor nodes detect events and report back to the basestation. The occurrence of the events can be sporadic and irregular in nature. The basestation is secure and extremely powerful when compared to the deployed sensor nodes. SPENA can be used for many applications requiring source node privacy. One class of applications is for tracking species of endangered animals or birds. Entities of such species need to be protected from hunters and poachers as they have great market value while at the same time they need to be studied. We consider an application in which the sensor network is deployed in a forest for sensing endangered birds (e.g. bald eagle). It is a homogeneous network, except for a secure basestation, and consists of light weight sensor nodes dispersed over a large area. Nodes sense the environment for the presence of endangered bird(s), and on detecting the same, report back to the basestation. Multiple sensor nodes can detect the event and will independently report it back to the basestation. Aggregation of the data occurs at the basestation.

The basestation gathers the information and is able to correlate it to identify the presence of the tracked entity, while also being able to study the flight patterns and their nesting habits. Given the tracked entity being swift and airborne, we can have multiple sensors detecting the event in a short period of time, and then not having any detection for a prolonged duration of time. The assumption is that, if the bird swooped down at a particular location, it did so to either prey or to get water, and this increases the possibility of the bird descending to that location thereby requiring source location privacy (secrecy) for the event reporting packet. In some applications the event can be sporadic, but it must be noted that 
there is still other communication between the sensor nodes, resulting in the generation of packet traffic.

\section{B. Threat Model}

The adversary is a super-local eavesdropper, and inconspicuously eavesdrops over a sensor network. By super-local eavesdropping capability, we mean the adversary can overhear communication at much larger distances compared to a sensor node. This overhearing radius can be up to 5 times that of a regular sensor node, there by providing the adversary a much larger overhearing area (up to 25 times) as compared to the sensor node. It also possesses the ability to compromise nodes. The adversary can be a single entity or a malicious network in itself, that senses over the deployed sensors. The adversary can operate in two modes:

1. Super-local eavesdropper: The adversary in this mode is passive in nature, and can listen to the packet being transmitted over a large area in the network. The adversary can either have a very powerful transceiver to overhear the communication or may have a network of its own to achieve eavesdropping. As a super-local eavesdropper, the adversary overhears the communications and can correlate the transmission of the packet over multiple hops. Although, the adversary cannot ascertain the contents of the packet when it eavesdrops over the transmission, it has the capability to compare two packets.

2. Stealth mode: An attacker in the stealth mode can compromise a node and get access to all the cryptographic information stored in the node. In this mode, the adversary takes control over the sensor node, and can decode the packet, and get information as available to any rightful sensor node. The adversary can either compromise a certain percentage of nodes randomly selected from the network or can compromise nodes which are geographically close to each other. Another form would be to compromise neighboring nodes.

The goal of the adversary is to identify the location and time of event occurrence, either by passive super-local eavesdropping or using intrusive node compromise. Alternately, the adversary may employ both. The problem is to conceal the event occurrence location and time by protecting source privacy under an eavesdropping and node compromise attack.

\section{SPENA SCHEME}

SPENA is a source privacy protection scheme which uses one-way hash chains and mapping functions. We introduce the basic idea in the preliminaries subsection, and then present the details of the scheme in the second subsection.

\section{A. Preliminaries}

In SPENA, a one-way hash chain is used to hide the source information. A one-way hash chain is a series of hash values generated by a one-way hash function. A basic idea of our approach is as follows. First, a unique hash function is used to generate a hash for source identification. This function is available at the source node and the basestation. Second, dynamically selected intermediate nodes on the routing path alter the packet resulting in a change to the packet structure.
Altering the packet structure disorients the packet traceback by the adversary. This modification of the packet structure is done such that the basestation can verify the information and connect it to the source generating the same.

\begin{tabular}{|l|l|l|c|c|l|l|}
\hline DstID & SrcID Hash & $\begin{array}{c}\text { Obfuscating } \\
\text { Partial Hash }\end{array}$ & $\begin{array}{c}\text { Rehash } \\
\text { Seed }\end{array}$ & $\begin{array}{c}\text { Payload } \\
\text { Length }\end{array}$ & Payload | SrclD & Filler \\
\hline
\end{tabular}

Fig. 1. Event Packet

SPENA can be used with a single path routing or a flooding based routing method. The event packet structure in SPENA is presented in Figure 1, and it has the following parameters:

1) DstID (Destination-id): It is the destination id of the packet. This is basestation for event packets.

2) SrcID hash (Source-id Hash): A unique hash of the source that is used to identify it at the basestation.

3) Obfuscating partial hash (OPH): This is generated by the source using the same hash function used to create the SrcID hash, and will be modified by dynamically selected intermediate nodes.

4) Rehash seed: Used to determine the intermediate nodes to reconstruct the packet en route to the basestation.

5) Payload Length: The length of a payload in the packet.

6) Payload: Payload is the actual data transmitted in the packet. It is encrypted using the symmetric key shared by the source node and the basestation. In the application presented in Section III, the payload consists of start time and duration of event occurrence.

7) Filler: Filler is used to provide a standard length to the packet and is populated with random garbage data. Having a constant packet size is required to prevent the adversary from tracking the packet based on increase in packet length during packet transformation. Keeping all the packets the same length forces the adversary to consider all packets while tracking back, and it cannot discount any packet analysis requirement based on the packet length. Using a garbage filler marginally increases the overhead, but the overhead is still very less compared to existing random walk or fake packet generation schemes as shown in Section V-C.

In the pre-deployment phase, each node is bootstrapped with four functions and a symmetric key. A hash function to generate the one-way hash chain, a second one-way hash function, a mapping function, and a rehash function. The entries from the hash chain are used as SrcID hash and the obfuscating partial hash as depicted in Figure 1. The hashes (SrcID hash and obfuscating partial hash) perform two critical functions. First, they provide privacy to the packet by preventing an adversary from performing a traceback to the source. Second, they help the basestation to check the validity of the packet received. Additionally, two event reporting packets from the same source will have different SrcID hash, protecting the network from an adversary by preventing the source of the two packets to be correlated. The second one-way hash function and the mapping function are used to select the intermediate 
nodes for modifying the packet. These selected nodes use the rehash function to hash the OPH into a fixed length, which is then encrypted using the key. Additionally, the rehashing node concatenates the SrcID hash from the received packet to the payload which is then encrypted using the symmetric key of the rehashing node. It updates the payload length field with the new payload length and subtracts the corresponding amount of bits from the filler field so as to maintain the standard packet size. The new filler field is filled with random garbage bits. The rehashing node replaces the SrcID hash with the hash from its hash-chain. The symmetric key is unique and known only to the basestation and the sensor node.

\section{B. SPENA Scheme}

As introduced earlier, SPENA uses a one-way hash function to hide the source information, a rehashing scheme to dynamically select the intermediate nodes for altering the packet, and finally, packet reception and verification at the basestation. These steps are explained in the following subsections.

1) One-way hash function: One-way hash function is attractive to use in sensor networks, because the values can be verified in a fraction of the time as compared to a digital signature. Given the resource constraints in sensor networks, we use the light chain method to generate the hash chain as described in [1]. Light chain generates a smaller hash when compared to the other methods. This is advantageous to use in sensor network given the limitations on the packet size and helps reduce the communication overhead. It can be further truncated without reducing the effectiveness since we use two hash values, the SrcID hash and the OPH. It should be also noted that, as in all hash chain usages, we use it in the reverse order and once the elements are exhausted, the hash is reset by the basestation. This is easily performed since the basestation keeps track of the hash value usage. When the hash chain entries near exhaustion (less than 10\% of entries are unused), the basestation resets it by transmitting a new seed to the sensor node. This packet is encrypted using the unique key shared by the sensor node and the basestation. This procedure consumes energy and will dictate the number of hash entries to be stored at the senor node. The number of entries stored at the sensor node has a tradeoff with the frequency of hash chain reset.

Hash-chain usage: Hash chain in SPENA is used for source identification and creating packet obscurity by certain randomly selected nodes on the packet path. This is different from the traditional sense of hashing that is primarily used for checking packet integrity in the form of a message digest.

Consider a network of $n$ sensor nodes. $H_{i}()$ is the one-way hash function for node $i$, generating a sequence of values $h_{i}^{1}$, $h_{i}^{2}, \ldots$ etc. When node $i$ wants to send an event packet to the basestation, and has already utilized $m-1$ entries from the one-way hash chain, it generates a packet shown in Figure 2, where $h_{i}^{m}$ is the $m^{t h}$ entry in the hash chain of node $i$.

The entry $h_{i}^{m}$ (SrcID hash) and $h_{i}^{m+1}$ (obfuscating partial hash) are consecutive entries generated by the hash function.

\begin{tabular}{|l|l|l|l|l|l|l|}
\hline DstID & $h_{i}^{m}$ & {$\left[R\left(h_{i}^{m+1} \mid \text { Payload }\right)\right]_{K_{i}}$} & $\begin{array}{c}\text { Rehash } \\
\text { Seed }\end{array}$ & $\begin{array}{c}\text { Payload } \\
\text { Length }\end{array}$ & {$[\text { Payload } \mid i]_{K_{i}}$} & Filler \\
\hline
\end{tabular}

Fig. 2. SPENA Event Packet

The hash value $h_{i}^{m}$ identifies the source at the receiver (basestation). A hash size of 4 bytes is used to prevent collisions while usage of smaller hash size is discussed later in the section. $h_{i}^{m+1}$ is used for obscuring the packet during its traversal by dynamically selected nodes along the packet route and also functions as a message authentication code for the packet. The source modifies the $h_{i}^{m+1}$ to encode payload information in the obfuscating partial hash where $R$ is the rehash function and $K_{i}$ is the key for node $i$ as seen in the third field in Figure 2.

2) Intermediate packet transformation: The intermediate packet transformation occurs at selected nodes on the path of the packet en route to the basestation. It has 2 steps: Selection of rehash nodes, and packet transformation.

Selection of rehash nodes: The random selection of rehashing nodes on the packet path is done using the rehash seed and a set of two functions: a one-way hash function and a mapping function. The functions are set in the node during pre-deployment bootstrapping, while the seed is read from the packet. This one-way hash function is different from the one used to generate the SrcID hash and the obfuscating partial hash. This method was used in [14] to generate random checkpoints and shown to be feasible to use in sensor networks.

For a node $j$, the one-way hash function is represented as $F_{j}(x)$, where $x$ is the rehash seed. The mapping function is $f_{\rho}(y)$, where $\rho$ is the rehash probability and $y=F_{j}(x)$. The mapping function has a range of $\{0,1\}$. For the range of output from the hash function, the mapping functions maps to 1 with probability $\rho$ and to 0 with probability $1-\rho$. Such a system of using two functions is beneficial because it gives the basestation a simple way to change the rehash probability by just replacing the mapping function using a broadcast. When a node $j$ receives a packet, it calculates $f_{\rho}\left(F_{j}(x)\right)$, and if the result maps to value 1 , it modifies the packet before forwarding to the next hop. If the mapping function maps to 0 , it forwards the packet unmodified. The number of intermediate nodes selected for rehashing is set by the basestation using the rehash probability parameter $\rho$. Additionally a rehashing node will hold the packet introducing a random delay of $d$ time units. This delay forces the adversary to consider all the packets that passed through the node in the $d$ time units while tracing back since the packet is reconstructed.

Packet transformation: The intermediate packet transformation occurs at nodes where the mapping function maps to a value of 1 , as described in selection of rehash nodes. Here, the rehashing node $j$ saves a copy of the packet before applying the transformation to it as follows:

1) SrcID hash: The value is replaced by a SrcID hash corresponding to rehashing node $j$.

2) $\mathrm{OPH}: \mathrm{OPH}_{j}=\left[R\left(O P H_{\text {Packet }}\right)\right]_{K_{j}}$ where $O P H_{\text {Packet }}$ is the obfuscating partial hash read from 
the packet, $R$ is the rehash function and $K_{j}$ is the key shared between the node $j$ and basestation.

3) Payload: Concatenate the SrcID hash received in packet to payload and encrypt with the symmetric key of node j. Payload Pay $_{j}=\left[R\left(\text { Payload }_{\text {Packet }} \mid h_{i}^{m}\right)\right]_{K_{j}}$, where Payload $_{\text {Packet }}$ is the payload of the received packet, $h_{i}^{m}$ is the SrcID hash of the received packet. Additionally, the network can decide to concatenate only partial SrcID hash to the payload in an attempt to reduce the overhead. This can lead to collisions, which can be deciphered by the basestation with the knowledge of network topology.

4) Payload Length: Length of the new payload.

5) Filler: Reduce the size of the filler to accommodate for the increased payload size and fill with garbage data.

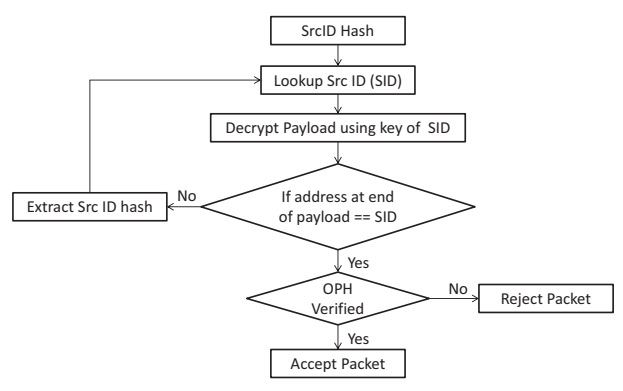

Fig. 3. Packet Reception and Verification

3) Packet reception and verification: On receiving a valid packet presented in Figure 2, the basestation uses a sliding window approach to look for the source id corresponding to the SrcID hash within the sliding windows of the hash table maintained for each node. A sliding window approach helps improve the search efficiency since the basestation does not need to go through the whole hash table. The length of the sliding window is governed by the packet loss rate in the network. The larger the packet loss rate, the larger this window is. When a valid packet is received, the starting point of the window is moved beyond SrcID hash in the table corresponding to the source.

The basestation, on receiving the packet does a reverse lookup on the SrcID hash to identify the source of the packet. We assume that the packet has undergone at least one rehash transformation during its traversal. It applies a recursive process as depicted in Figure 3, until it reaches the true source id of the packet. During this process the basestation identifies the intermediate nodes responsible for rehashing the obfuscating partial hash. With this knowledge, the basestation applies the rehashing process to the value of 'obfuscating partial hash' starting with the hash value as encoded by the source. If the basestation reaches the same value as received in the packet, this verifies the integrity of the packet received.

\section{AnAlysis and Evaluation}

We present the analysis for SPENA, and evaluate the protocol overhead and compare it with other existing methods.

\section{A. Security Analysis}

To check SPENA's ability to withstand different attacks, the adversary is provided with varying capabilities and we measure the effectiveness of SPENA against each form of the adversary. For this, we consider the adversary to be in super-local eavesdropper mode and then give it additional capabilities of node compromise in super-local eavesdropper stealth mode. The notations used are listed in Table I.

TABLE I

LIST OF NOTATION

\begin{tabular}{|ll|ll|}
\hline$n$ & Number of nodes & $d$ & Delay introduced by rehashing node \\
\hline$l$ & Average path length & $e$ & Number of rehash nodes on path \\
\hline$c$ & Total compromised nodes & $p$ & Packet generation rate \\
\hline$\rho$ & Rehash probability & $v$ & Rehashing node address size \\
\hline
\end{tabular}

1) Super-local eavesdropper: A super-local eavesdropping adversary can overhear packet communication over a large part of the network. The adversary can compare two packets as string length of bits, but it cannot decrypt the contents of the packet. Also, the adversary can compare packets (same packet over different hops) and identify if the packets are the same. When packet traversal occurs from source to the destination, the adversary can overhear the packet transmission and verify if the packet transmitted over different hops are the same.

In SPENA, we use a rehash node selection method, which dynamically selects the rehashing nodes on the packet path, and the number of selected nodes is determined by the rehash probability parameter $(\rho)$. These selected nodes modify the packet structure by reconstructing the SrcID hash, OPH, payload, filler, and payload length fields while maintaining the same packet length. This results in the packet received and the packet forwarded by the node seem different to the eavesdropper. Hence, while tracing back, the adversary cannot follow the packet path without considering all the packets generated. It should be noted that in addition to the event packets, there will be other communication among the nodes resulting in generation of a large number of packets, which makes it costly for the adversary to perform detailed analyses on each hop. Consider a network of size $n$ with an average path length of $l$ hops. Given the rehash probability parameter $\rho$, the number of intermediate nodes selected for rehashing is $l \cdot \rho$. The packet generation rate is $p$ packets per node per unit time, giving the total number of packets generated in the network in a unit time as $p \cdot n$. The number of packets generated over the period of time over $l$ hops with each hop utilizing one unit time is $p \cdot n \cdot l$. For the duration of $l$ hops, the number of packets generated, considering each rehashed packet as a different packet is $(n \cdot p \cdot l)\left(1+\frac{\rho(l+1)}{2}\right)$.

Under the circumstances of doing an in depth packet analysis, the adversary will have to compare the packet with $(n \cdot p \cdot l)\left(1+\frac{\rho(l+1)}{2}\right)$ packets considering no additional delay is introduced by the rehashing nodes. For a network of 500 nodes, the number of packet comparisons required for varying path lengths and different rehash probability is presented in Figure 4. The packet generation rate is 0.1 , and the rehash probability is 0.1 . 


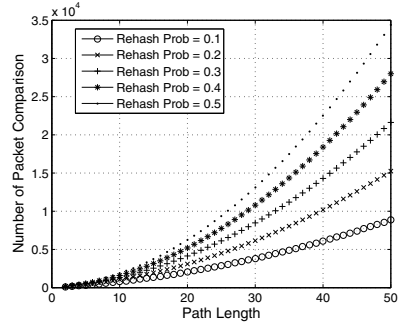

Fig. 4. Packet Comparison

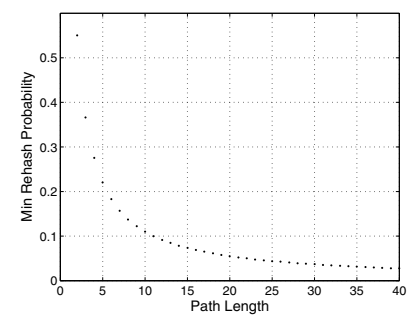

Fig. 5. Minimum Rehash Probability
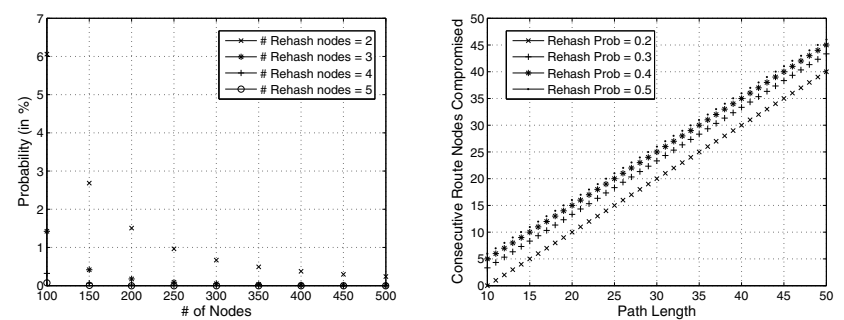

Fig. 6. Probability of Compromising Fig. 7. Consecutive Route Nodes all Rehash Nodes

Compromised
If $d$ is the random delay introduced, the average number of packets forwarded by each node is $d \cdot p \cdot l$. The adversary will now need to consider each of the source of $d \cdot p \cdot l$ packets as a potential sender of the packet. If it randomly selects a neighbor to be the sender, then the probability of selecting the valid sender is $\frac{1}{q}$, where $q$ is the number of neighbors forwarding packets. On selecting the valid neighbor, it needs to consider all the packets that were transmitted from the neighbor to the node in the last $d$ time units i.e. $d \cdot p \cdot l$. This gives an overall probability of tracking the packet back as $\left(\frac{1}{d \cdot p \cdot l \cdot q}\right)^{\rho \cdot l}$. The higher the delay, the larger is the set of packets required to be analyzed, but the drawback being the increased cumulative delay introduced in packet delivery at the basestation.

A network with a long path $l$ or a big neighborhood is beneficial to our scheme. Hence, either a network with a long path length $l$ or a dense network with a big neighborhood, which are both characteristics of a sensor network bodes well to our scheme. Also, high packet generation rate reduces the probability of a successful traceback. In a random sensor network deployment, the factor under the control of the basestation is the rehash probability, which can be increased to reduce the probability of a successful traceback. It should be noted that the basestation is significantly more powerful than a sensor node and with advent of technology, it can handle a very high packet rate even in a large network.

Impact of $\rho$ : The higher value of $\rho$ results in increased processing by the intermediate nodes. This can add to energy consumption. Additionally, it will have an impact on the length of address addition to the packet as each rehashing node encodes the source id hash from the packet received. However, the higher $\rho$ value leads to increased selection of intermediate rehashing nodes, thereby obfuscating the packet further. We need at least one node to be selected as a rehashing node, hence based on the value of $l$, we have a minimum requirement for $\rho$. Taking into consideration an error of $10 \%$, the minimum $\rho$ value for different path lengths is depicted in Figure 5. It should be noted that the probability of successful communication is not dependent on $\rho$, but on channel charateristics and is the same as in a communication without rehashing.

2) Stealth mode: In the stealth mode, the adversary is a powerful entity that can compromise a set of sensor nodes. We analyze if the access to cryptographic information due to node compromise allows an adversary to identify the original source of the packet when using SPENA. The adversary has access to all the functions and the keys of the node. On receipt of the packet at the compromised node, it can read the entire packet and scrutinize each component of the packet. We begin by analyzing the access to each component in the packet.

1) DstID: Readable and is available in plain text.

2) SrcID hash: This is a hash value generated by a oneway hash function, and unless the source itself, or the basestation is compromised, this information is secure. Additionally, this field changes when it is updated to the source-id hash of the selected rehashing node.

3) Obfuscating partial hash: This is again a hash value generated by the one-way hash function, which may have been rehashed en route to the compromised node. Access to this hash value in its true form (as encoded by the source) or the rehashed form does not leak any information about the source.

4) Rehash Seed: The seed value can be used by the adversary to identify if it needs to rehash the packet and has no correlation to the source generating the packet.

5) Payload Length: It identifies the length of the payload and does not leak any other information.

6) Payload: It is an encrypted field and without access to decryption keys does not disclose any information.

7) Filler: The filler is garbage data which is changed at each rehashing node.

A compromised node can belong to one of the three types, an intermediate node, an intermediate rehashing node, or a source node. The function of an intermediate node is to forward the packet as it is. An intermediate node compromise does not yield any additional information when compared to the information already gained by eavesdropping. When the intermediate node is a rehashing node, the adversary gains the knowledge of the incoming packet and its transformed next hop. By itself, this does not provide any advantage, but when used with eavesdropping this is useful to the adversary. A compromise of all rehash nodes on the packet path will allow the adversary to traceback the packet while performing eavesdropping. Access to the key and the functions at the compromised node will not allow the adversary to decode the passing packet since the payload is encrypted using the symmetric key of the source node and the source-id is hidden in the form of a hash value and can be mapped to the source 
node only by the basestation. The last case, i.e., compromise of the source node will reveal the source information. We see that, just compromising the nodes does not give the adversary much advantage unless the source node is compromised, but when done in addition to eavesdropping, it becomes a useful tool. So, further analysis of stealth mode is done in addition to the adversary having eavesdropping capability.

3) Super-local eavesdropping and stealth mode: This is the most advanced form of attack in which the adversary eavesdrops over a large portion of the network with ability to compromise nodes and access the cryptographic information.

The method of selecting the compromised nodes is categorized into three types. In the first method, the compromised nodes are randomly selected by the adversary and they can be dispersed anywhere in the whole network. In the second case, the compromised nodes are bound to a geographical area and the nodes are assumed to be interconnected. This can occur if the adversary plans on compromising nodes by picking a neighboring node of the last compromised node. Thirdly, the adversary can compromise all available sensor nodes within a given radius. This is the easiest scenario, but is less plausible, since the adversary may not apply its resources to perform such an attack unless it is absolutely certain of the origination of the packet in the geographical area.

1. Random node compromise: In random node compromise, the adversary selects $r \%$ of nodes to be randomly compromised. Random node compromise is employed by the adversary when it lacks the ability to intelligently select nodes to compromise. Given the network of size $n$, the number of compromised nodes on a given packet route is $\frac{c \cdot l}{n}$, where $c$ is the total number of compromised nodes given by $\frac{r \cdot n}{100}$. This gives us the number of compromised nodes on a path as $\frac{r \cdot l}{100}$. Let's analyze the worst case scenario when all the rehash nodes on a packet path are compromised because, the only case for an adversary to do a successful traceback is when all the rehash nodes are compromised. If the number of rehash nodes on the packet path is $e$, then the probability of selecting all $e$ rehash nodes is $\left(\begin{array}{l}c \\ e\end{array}\right) /\left(\begin{array}{l}n \\ e\end{array}\right)$. An example is depicted in Figure 6, which shows the probability of an adversary selecting all the rehash nodes under different network sizes given the number of compromised nodes is 25 .

It is noted that as the number of nodes grows larger than a few hundred, the probability of all rehash nodes being compromised decreases significantly. For example, the probability of 3 rehash nodes compromised in a 400 node network when 25 randomly selected nodes are compromised is 0.00021 . Also, with the increase in the number of rehash nodes, this probability value decreases further.

2. Compromise neighboring nodes: The $r \%$ of nodes compromised belong to a neighborhood. By neighborhood, we imply that the nodes can all reach each other, albeit over multiple hops. When considering a path of length $l$, the worst case scenario occurs if all the $c$ compromised nodes belong to the same path. We are interested in the cases where $c<l$, because if $c \geq l$, then the source itself is compromised and it is not possible to protect its privacy. With $\rho$ as the rehash probability, the traceback will be unsuccessful as long as $c<l-2 \cdot \frac{1}{\rho}$. Figure 7 depicts the minimum number of consecutive nodes on a route that needs to be compromised for different path lengths with varying rehash probabilities.

A neighboring node compromise scenario is used by the adversary with the hope that sufficient consecutive nodes on a given path are compromised to reveal the source information. Another case in point is to compromise nodes surrounding an area so as to either track packets originating from the area (source tracking) or track packets to a particular destination.

TABLE II

ATtACK Distribution

\begin{tabular}{|c|c|c|}
\hline Attack radius & Avg Compromised Node & Max Compromised Node \\
\hline 13 & 1.17 & 5 \\
\hline 25 & 1.6 & 8 \\
\hline 50 & 4.19 & 13 \\
\hline
\end{tabular}

3. Compromised nodes in a geographical area: Compromising nodes in a geographical area is employed when the adversary estimates the presence of the source node in the area. To ease the analysis we assume the geographical area to be circular with an attack radius equal to transmission range $t$. The number of compromised nodes will vary with the attack radius. Lets consider a sensing range of $25 \mathrm{~m}$. To have a unit coverage over a 1000x1000 area, we need 509 nodes. Simulation results of the average compromised nodes and maximum compromised nodes are presented in Table II.

From Table II, a very uneven distribution with the node compromise area being densely populated is required for such a scenario. If all the rehashing nodes on the packet path are compromised, with super-local eavesdropping overhearing over the whole network path, the adversary will be able to trace the packet back to the source. Hence, there needs to be at least one rehashing node that is not a compromised node. The distance between the first and the last rehashing node on the path is $l-2 \cdot \frac{1}{\rho}$. In the worst case scenario where all the compromised neighbor nodes are on the path, if $\left(l-2 \cdot \frac{1}{\rho}\right)>a$ (where $a$ is the longest path possible within the compromised geographical area), then the source information is secure, since at least one of the rehashing nodes is still un-compromised.

\section{B. Overhead Analysis}

It is accepted that any inclusion of security principles will incur additional overhead. It needs to be seen if the overhead incurred is within reasonable limits and whether the trade off against increased energy consumption and delay is justified.

We assume the standard 36 byte packet. Instead of the source id, we use a hash generated by a one-way hash function with the SrcID hash equal to 4 bytes instead of the regular 2 bytes. The obfuscating partial hash can be either 1 or 2 bytes as dictated by the basestation. This obfuscating partial hash can be any pre-decided part of the 4 byte hash generated by the one-way hash function. The reason it can be short is because it is used in addition to the SrcID hash for packet validation purposes and doubles as a message authentication code. The rehash seed and the payload length together are 1 byte long. 
Hence, in SPENA, an event packet uses 4 additional bytes when compared to a regular sensor packet of size 36 bytes.

SPENA also uses additional space for the intermediate nodes to program the SrcID hash information into the payload. For this purpose, we use a partial source hash and not the full 4 bytes to conserve overhead and trade off a small percentage of accuracy, in the case where the partial hash can belong to more than one node. Under these circumstances, the basestation makes an estimate of the rehashing node based on the geographical location. The space reserved for the address will depend on the number of rehashing intermediate nodes selected. Also, it should be noted that the payload in an event packet for an application like tracking for endangered birds is small, hence allowing the rest of the packet to be used for header. So, to improve the accuracy, the basestation can decide on allowing the intermediate rehashing nodes to include a larger partial address hash. For applications requiring larger payload, the data can be split and transmitted in multiple packets, thus preserving the accuracy (with larger hash sizes) but increasing the overhead. If $v$ is the size of the intermediate rehashing node address size, we have the overhead as $\frac{4+v \cdot l \cdot \rho}{36}$.

The rehashing nodes introduce a random delay of $d$ units. The intermediate node checking for rehash variability takes minuscule amount of time compared to the delay introduced. On a $l$ hop path with rehash probability of $\rho$, the total time taken for the packet to traverse its path is $l(1+\rho \cdot d)$. The delay parameter can be set by the basestation based on the applications real-time requirements.

The storage of the hash chain entries in the sensor node consumes memory. If we consider a standard IRIS mote with $128 \mathrm{~KB}$ program flash memory $(512 \mathrm{~KB}$ measurement flash memory), then to store 128 hash chain entries takes $512 \mathrm{~B}$ of memory. Even if we consider 250 hash chain entries, it takes less than $1 \%$ of the program flash memory. Additionally, the number of hash chain entries to be stored in a node can be decided by the basestation. A smaller number of entries will require the basestation resetting the chain more frequently which consumes transmission energy. Hence, based on the application, the basestation needs to decide the chain length.

Next, we consider the additional processing occurring at each node. It is noted that transmitting $1 K b$ of data over $100 \mathrm{~m}$ consumes the same amount of energy as executing 3 million instructions on a processor with $100 \mathrm{MIPS} / \mathrm{W}$ power [9]. Kaps, et al. [8] measured the energy consumed to encrypt a packet using SHA-1 as $43.32 n J$, while the total energy to transmit a packet at maximum power is $93.3 \mu \mathrm{J}$, making transmission costlier by an order of 3 . This makes the intermediate node processing energy consumption insignificant when compared to the communication cost incurred. Also, only a few nodes are involved in the packet reconstruction process, while the other nodes only use the rehash seed to check if they are selected as a rehashing node for this packet.

The number of address lookups, packet verification will put an additional load on the basestation. If we consider the basestation to be running on a standard server configuration, having a quad-core CPU, with $8 \mathrm{~GB}$ of RAM, it should be able to administer any amount of packets generated within practical limits. If the sensor network grows beyond a certain size, then multiple basestations can be used, allowing implicit load balancing while also conserving the resources of the intermediate sensor nodes (reducing packet propagation overhead).

\section{Comparison}

We compare SPENA with DCARPS [5], Random Walk [2], and fake event packet generation [4] [11]. DCARPS uses label-switching method, while SPENA uses a hash chain and intermediate packet altering scheme to hide source traversal information. SPENA is lightweight and makes much less assumptions while considering a strong threat model. DCARPS requires the basestation to have knowledge of the topology of the network, and our work makes no such assumption. The basestation creates a routing tree and routing paths in DCARPS and is relayed to the nodes. SPENA has no such requirements and has the advantage of being set to work with single path routing as well as a flooding based protocols. In DCARPS, all the nodes on the path need to decrypt and reencrypt the packet with a new label, while in SPENA, only a fraction of the nodes are required to reconstruct the packet before forwarding. Additionally, in SPENA, this reconstruction is done such that it allows the basestation to validate the packet, whereas in DCARPS it is only used for routing. Node compromise attacks are not considered in DCARPS.

Comparing SPENA to technique involving random walk [2]. The length of the random walk determines the distance from the source to which a local eavesdropper can traceback. When you consider an eavesdropping adversary, then a random walk scenario fails, since the eavesdropper can traceback the packet to the source through the random walk. The overhead involved in random walk is higher than SPENA and is presented in Figure 8 . The random walk length values of $10,15,20$, and 25 are the number of random hops over which the packet propagates before moving towards the basestation. We consider the rehashing value of 0.1 for SPENA, and the filler is such that it compensates for the largest path size. We consider the longest path length of 40 hops. Thus, the number of rehashing nodes is 4 , requiring a filler of size 8 bytes to maintain a fixed packet size even after transformation. This gives us an overhead of 0.34 , i.e., the total additional overhead required for SrcId hash, OPH, rehash seed and payload length, rehashing node address (total 12 bytes) over 36 bytes when the intermediate node incorporates a 2 byte hash address in the payload. We define privacy in random walk based methods as the length of distance the random walk takes the source information away from the actual source when compared to the path length. Figure 9 presents the privacy achieved for the same path lengths and random walk lengths as presented in Figure 8. The overhead is the resources (energy in our case) consumed due to propagation over the random path before the packet starts its journey towards the basestation. As the overhead is reducing for random walk, the privacy achieved also reduces as in Figure 9 while the overhead of SPENA is constant irrespective of the path lengths. 


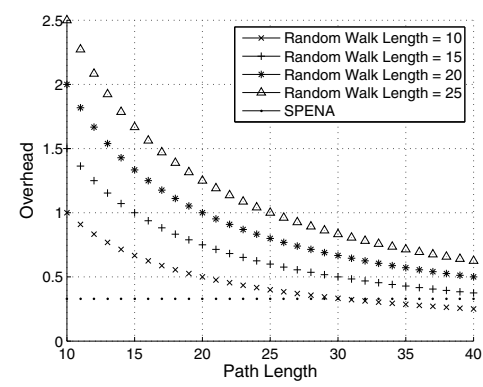

Fig. 8. Random Walk Overhead

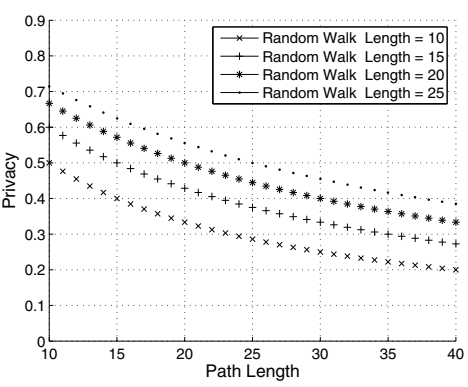

Fig. 9. Random Walk Privacy

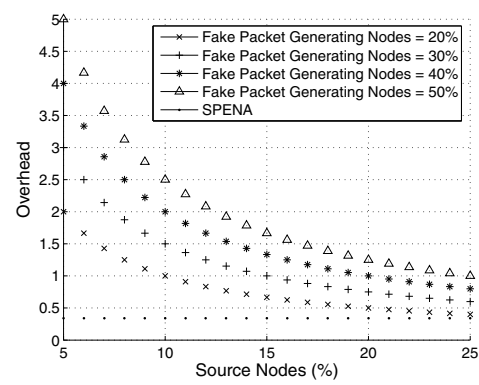

Fig. 10. Fake Packet Generation Overhead
The other methods using fake packet generation [4] [11] by nodes to emulate a packet introduce extremely large overhead. This is an obfuscating technique and is only successful under heavy load of fake packet generation. If one node detects an event and generates the event reporting packet, there needs to be at least one more source node generating a fake event reporting packet. Under these circumstances we see that, of all the traffic generated, $50 \%$ of the traffic corresponds to fake traffic. Furthermore, to increase security will incur more overhead in the form of fake packets. Hence, the valid functionality of such a system is dependent on the amount of fake packets generated and the scenario providing very minimal source privacy still has more overhead than SPENA. Additionally, the fake packet generation scheme does not consider node compromises.

Figure 10 presents the overhead incurred in fake packet generation compared to SPENA. We consider different numbers of fake packet generating nodes, with the number ranging from $20 \%$ to $50 \%$ of all nodes. The 'source node (\%)' on $x$ axis corresponds to the number of actual source nodes in the network as a percentage of all nodes. It is seen that any form of fake packet generation technique will have significantly higher overhead compared to SPENA. Even the conservative case of having just $20 \%$ of sensor nodes generating fake packets results in higher overhead compared to SPENA.

\section{CONCLUSION}

Source privacy of the sensor node generating the event is important as it can give away the event occurrence location. Existing techniques consider an eavesdropping adversary and refrain from providing source privacy solutions to an intrusive node compromise attack with eavesdropping. In this work, we first present a hash-chain based source information hiding scheme. Second, a packet reconstruction strategy by dynamically selected intermediate nodes on the packet path, which can protect source privacy under eavesdropping attacks while also tolerating node compromise attacks, is presented. Third, using the same one-way hash chain function, we provide a packet verification method for the basestation to validate a received packet. Finally, we analyze our protocol under different attack scenarios, and evaluate its efficiency in terms of overhead when compared to existing work.

\section{ACKNOWLEDGEMENT}

This work was supported in part by the US National Science Foundation under Grants CCF-0514078, CNS-0551464, and CNS-0721441.

\section{REFERENCES}

[1] Y.-C. Hu, M. Jakobsson, and A. Perrig. Efficient constructions for oneway hash chains. In Applied Cryptography and Network Security, pages 423-441, 2005.

[2] P. Kamat, Y. Zhang, W. Trappe, and C. Ozturk. Enhancing sourcelocation privacy in sensor network routing. In Proceedings of the 25th IEEE International Conference on Distributed Computing Systems, Washington DC, 2005.

[3] C. Karlof, N. Sastry, and D. Wagner. Tinysec: a link layer security architecture for wireless sensor networks. In Proceedings of the 2nd international conference on Embedded networked sensor systems, pages 162-175, New York, USA, 2004. ACM.

[4] K. Mehta, D. Liu, and M. Wright. Location privacy in sensor networks against a global eavesdropper. In Proceedings of the IEEE International Conference on Network Protocols (ICNP 2007), Oct. 2007.

[5] A. A. Nezhad, A. Miri, and D. Makrakis. Location privacy and anonymity preserving routing for wireless sensor networks. Computer Networks, 52(18):3433 - 3452, 2008.

[6] Y. Ouyang, Z. Le, D. Liu, J. Ford, and F. Makedon. Source location privacy against laptop-class attacks in sensor networks. In Proceedings of the 4th international conference on Security and privacy in communication netowrks, pages 1-10, New York, USA, 2008. ACM.

[7] A.-S. K. Pathan and C. S. Hong. Serp: secure energy-efficient routing protocol for densely deployed wireless sensor networks. Annales des Télécommunications, 63(9-10):529-541, 2008.

[8] J. peter Kaps and B. Sunar. Energy comparison of aes and sha-1 for ubiquitous computing. In IFIP International Conference on Embedded and Ubiquitous Computing (EUC 2006), LNCS. Springer, 2006.

[9] G. J. Pottie and W. J. Kaiser. Wireless integrated network sensors. Communications of ACM, 43(5):51-58, 2000.

[10] T. Roosta, S. Shieh, and S. Sastry. Taxonomy of security attacks in sensor networks and countermeasures. In The First IEEE International Conference on System Integration and Reliability Improvements, Hanoi, Vietnam, December 2006.

[11] M. Shao, Y. Yang, S. Zhu, and G. Cao. Towards statistically strong source anonymity for sensor networks. In INFOCOM 2008, The 27th Conference on Computer Communications, pages 51-55, April 2008.

[12] H. Wang, B. Sheng, and Q. Li. Privacy-aware routing in sensor networks. Computer Networks, 53(9):1512 - 1529, 2009.

[13] R. Watro, D. Kong, S.-f. Cuti, C. Gardiner, C. Lynn, and P. Kruus. Tinypk: securing sensor networks with public key technology. In SASN '04: Proceedings of the 2nd ACM workshop on Security of ad hoc and sensor networks, pages 59-64, New York, USA, 2004. ACM.

[14] B. Xiao, B. Yu, and C. Gao. Chemas: Identify suspect nodes in selective forwarding attacks. Journal of Parallel and Distributed Computing, 67(11): 1218 - 1230, 2007.

[15] Y. Yang, M. Shao, S. Zhu, B. Urgaonkar, and G. Cao. Towards event source unobservability with minimum network traffic in sensor networks. In WiSec '08: Proceedings of the first ACM conference on Wireless network security, pages 77-88, New York, NY, USA, 2008. 